\title{
Long-term sex-differential effects of neonatal vitamin A supplementation on in vitro cytokine responses
}

\author{
Kristoffer J. Jensen ${ }^{1,2,3 *}$, Mia J. Søndergaard ${ }^{2,4}$, Andreas Andersen ${ }^{1}$, Cesario Martins ${ }^{2}$, Christian Erikstrup ${ }^{5}$, \\ Peter Aaby ${ }^{2}$, Katie L. Flanagan ${ }^{6}$ and Christine Stabell Benn ${ }^{1,2,7}$ \\ ${ }^{1}$ Research Center for Vitamins and Vaccines, Bandim Health Project, Statens Serum Institut, DK-2300 Copenhagen, Denmark \\ ${ }^{2}$ Bandim Health Project, Indepth Network, Bissau, Bissau codex 1004, Guinea-Bissau \\ ${ }^{3}$ The National Veterinary Institute, Technical University of Denmark, DK-2800 Kgs Lyngby, Denmark \\ ${ }^{4}$ Department of Pediatrics, Nordsjaellands Hospital, DK-3400 Hillerød, Denmark \\ ${ }^{5}$ Department of Clinical Immunology, Aarhus University Hospital, DK-8000 Aarbus, Denmark \\ ${ }^{6}$ Department of Immunology and Pathology, Monash University, Melbourne, VIC 3800, Australia \\ ${ }^{7}$ Odense Patient data Explorative Network (OPEN), Odense University Hospital/Department of Clinical Research, University of \\ Southern Denmark, DK-5000 Odense, Denmark
}

(Submitted 9 March 2017 - Final revision received 23 July 2017 - Accepted 29 August 2017 - First published online 23 November 2017)

\section{Abstract}

High-dose vitamin A supplementation (VAS) may affect mortality to infectious diseases in a sex-differential manner. Here, we analysed the long-term immunological effects of neonatal vitamin A supplementation (NVAS) in 247 children, who had been randomly allocated to 50000 or $25000 \mathrm{IU}$ vitamin A ( $15 \mathrm{mg}$ and $7.5 \mathrm{mg}$ retinol equivalents, respectively) or placebo at birth. At 4-6 months of age, we assessed bacille Calmette-Guérin (BCG) scarification, and we analysed in vitro responses of TNF- $\alpha$, IL- 5 , IL-10, IL-13 and IFN- $\gamma$ in whole blood stimulations to phytohaemagglutinin (PHA), purified protein derivative (PPD), tetanus toxoid and lipopolysaccharide. There were no differences between the two doses of NVAS, and thus they were analysed combined as NVAS (any dose) $v$. placebo. All analyses were performed unstratified and by sex. NVAS increased the chance of having a scar after BCG vaccination in females (NVAS $v$. placebo: $96 v$. $71 \%$, proportion ratio: $1.24 ; 95 \%$ CI $1.09,1.42)$, but not in males $\left(P_{\text {for interaction }}=0.012\right)$. NVAS was associated with significant sex-differential effects on the pro- to antiinflammatory cytokine ratios (TNF- $\alpha$ :IL-10) to PPD, tetanus toxoid and medium alone, which were increased in females but decreased in males. In addition, IL-17 responses tended to be increased in NVAS $v$. placebo recipients in males but not in females, significantly so for the PHA stimulation. The study corroborates sex-differential effects of VAS on the immune system, emphasising the importance of analysing VAS effects by sex.

\section{Key words: Vitamin A supplementation: Infants: Cytokines: Heterologous immunity: Sex differences}

Vitamin A and its metabolites are essential for the functioning of the immune system ${ }^{(1)}$. Vitamin A supplementation (VAS) is recommended for children from 6 months to 5 years of age in populations with high risk of vitamin A deficiency (VAD) to reduce VAD-related morbidity and mortality ${ }^{(2)}$. High-dose VAS in the neonatal period (NVAS) is not WHO policy but has been tested in several randomised controlled trials (RCT) and is currently being considered as a policy, at least for sub-groups ${ }^{(3,4)}$. Our research group in Guinea-Bissau has conducted several of the NVAS trials, and found that NVAS had sex-differential effects on mortality, being associated with slightly lower mortality in males, but higher mortality in females ${ }^{(5-7)}$. The negative effect in females increased with increasing length of follow-up, and other studies have indeed corroborated this pattern; in all existing trials with follow-up to 12 months of age, females who had received NVAS had higher mortality than females who had received placebo at birth from 6 to 12 months of follow-up ${ }^{(8)}$. We have hypothesised that this could be due to a negative interaction between NVAS and subsequent diphtheria-tetanus-pertussis (DTP) vaccination (recommended in three doses at 6,10 and 14 weeks of age) in females ${ }^{(8,9)}$. This observation is epidemiologically founded. Explorative immunological studies may generate hypotheses, which could guide the design of further mechanistic studies. In the present explorative study, we took advantage of two existing trial cohorts to assess sex differences in the

\footnotetext{
Abbreviations: BCG, bacille Calmette-Guérin; DTP, diphtheria-tetanus-pertussis vaccine; GMR, geometric:mean ratio; MV, measles vaccine; NVAS, neonatal vitamin A supplementation; PPD, purified protein derivative from Mycobacterium tuberculosis; RCT, randomised controlled trial; VAS, vitamin A supplementation.
}

* Corresponding author: K. J. Jensen, email krijen@vet.dtu.dk 
immunological response to NVAS, which could help explain the sex differences in the mortality effects.

The early measles vaccine (MV) trial was a RCT testing the effect of providing an additional dose of early (i.e. 4-6 months of age) dose of MV in Guinea-Bissau, West Africa. Children were enrolled at the age of 4-6 months after the third dose of DTP and randomised to early MV or no early MV in addition to the usual MV at the age of 9 months (Fig. 1) ${ }^{(10)}$. Before randomisation, a blood sample was collected from a subgroup of the children for an immunological study ${ }^{(11)}$. Independent of the early MV trial, some participants had previously participated in an RCT of NVAS ${ }^{(6)}$. Using the blood samples collected as a baseline in the early MV trial (before allocation to early MV or no early MV) ${ }^{(11)}$, we analysed the association between NVAS and in vitro cytokine responses in the 4- to 6-month-old infants who had participated also in the NVAS trial.

\section{Methods}

\section{Setting}

The study took place at the Bandim Health Project in GuineaBissau (www.bandim.org), which follows a population of about 100000 people in a health and demographic surveillance system. The study was defined by two existing trial cohorts, involving a group of children who had participated in first an NVAS trial, and then, independent of the NVAS trial, participated in the early MV trial, including an immunological MV sub-group study ${ }^{(11)}$.

\section{Neonatal vitamin A supplementation trial}

From 2004 to 2008, an RCT of NVAS was conducted in the study area randomising normal-birth weight $(>2500 \mathrm{~g})$ infants 1:1:1 to receiving $15 \mathrm{mg}$ retinol equivalents (RE) (50 $000 \mathrm{IU}$ vitamin A), $7.5 \mathrm{mg}$ RE (25000 IU vitamin A) or placebo at birth; all received concurrently bacille Calmette-Guérin (BCG) vaccine ${ }^{(6)}$.

\section{Early measles vaccine trial}

From 2003 to 2007 , an RCT of early MV was conducted, testing the effect on mortality of giving an additional MV earlier than the generally recommended MV at 9 months of age ${ }^{(10)}$. In brief, infants who had received three scheduled DTP vaccinations were eligible for enrolment and randomisation to early MV (Edmonston-Zagreb) at 4-6 months of age or no early MV (all infants were to receive the recommended MV at 9 months). Thus, at enrolment, infants had DTP 3 + oral polio vaccine 3 as their last vaccines.

\section{Immunological measles vaccine sub-group study}

The immunological MV sub-group study to investigate the nonspecific immunological effects of MV (clinicaltrials.gov: no. NCT00168545) took place in 2006. From January through September 2006, immediately before randomisation to early MV or no early MV at 4.5 months of age, a physician examined children for eligibility. To avoid that acute illness influenced plasma biomarkers and stimulated cytokine production, children with the following characteristics were excluded from the immunological study: current fever or diarrhoea reported by the mother; an axillary temperature above $37.5^{\circ} \mathrm{C}$; a respiratory rate at $60 /$ min or above; or current infection diagnosed by the physician. BCG scarification was assessed and a blood sample was taken by finger prick. The present study is based on this baseline (preMV) sample from the early MV trial. Some children had a venous instead of capillary blood sample obtained, but we excluded venous samples because we found differences in the cytokine responses in venous and capillary blood ${ }^{(12)}$. Analytical details can be found in ${ }^{(11)}$. In brief, whole blood was diluted 1:9 with RPMI-1640 containing streptomycin $(100 \mathrm{mg} / \mathrm{ml})$, penicillin $\mathrm{A}(100 \mathrm{IU} / \mathrm{ml})$, glutamate $(2 \mathrm{~mm})$ and pyruvate (1 mm) (all Gibco). The diluted blood was stimulated with lipopolysaccharide (LPS, $1 \mathrm{ng} / \mathrm{ml}$; Sigma-Aldrich) (a Toll-like receptor (TLR)4 ligand), (S)-(2,3-bis-(palmitoyloxy)(2-RS)-propyl)- $N$-palmitoyl-(R)-Cys-(S)-Ser-(S)-Lys4-OH, trihydrochloride (Pam3CSK4, $100 \mathrm{ng} / \mathrm{ml}$; Cayla-InvivoGen Europe, a TLR2 ligand), phytohaemagglutinin (PHA, $2 \mu \mathrm{g} / \mathrm{ml}$; Wellcome Diagnostics), tetanus toxoid (TT, $1.5 \mathrm{Lf} / \mathrm{ml}$ ), purified protein derivative (PPD) from Mycobacterium tuberculosis $(10 \mu \mathrm{g} / \mathrm{ml}$; Statens Serum Institut) or medium alone. Concentrations of cytokines were measured from supernatants using the Luminex platform (Luminex 100; Luminex Corp.) (lower limit of detection (LLD) given in parenthesis): TNF- $\alpha$ (10 pg/ml), IFN- $\gamma$ $(5 \mathrm{pg} / \mathrm{ml}), \mathrm{IL}-5(3 \mathrm{pg} / \mathrm{ml}), \mathrm{IL}-10(5 \mathrm{pg} / \mathrm{ml}), \mathrm{IL}-13(10 \mathrm{pg} / \mathrm{ml})$ and IL-17 $(10 \mathrm{pg} / \mathrm{ml})$ (BioSource). In unstimulated plasma, concentrations of the following compounds were measured using the Luminex assay kit (Fluorokine MAP Multiplex Human Cytokine Panel A; R\&D Systems): TNF- $\alpha$ (LLD: 5 pg/ml), IL-10 $(5 \mathrm{pg} / \mathrm{ml})$, IL-6 (7 pg/ml), monocyte chemoattractant chemokine protein (MCP)-1 (10 pg/ml), IL-8 (2.8 pg/ml) and IL-1Ra (30 pg/ $\mathrm{ml}$ ); concentrations of soluble urokinase plasminogen activator receptor (suPAR) was measured by ELISA (suPARnostic standard ELISA, ViroGates) ${ }^{(11)}$.

Statistical methods have been described previously ${ }^{(11)}$. Cytokine data were log-transformed and analysed with Tobit regression using multiple imputations to account for measurements below the LLD of the assay, and estimates were backtransformed providing geometric mean ratios (GMR). Where $>50 \%$ of measurements were below the LLD of the respective analytes, Poisson regression was used, providing proportion ratios (PR) of measurements above LLD as done previously $^{(13,14)}$. A summary of the proportion of measurements $>50 \%$ and the geometric means can be found in the online Supplementary Table S1.

For distributions with $<50 \%$ observations below LLD, the ratios of IFN- $\gamma$ to IL- 5 , IFN- $\gamma$ to IL-10 and TNF- $\alpha$ to IL-10 were analysed and reported as GMR-ratios (GMRR).

Exploratory data analysis indicated that there was no difference in the effect of $7.5 \mathrm{mg} \operatorname{RE} v .15 \mathrm{mg}$ RE overall or stratified by sex; hence, the two doses were analysed collectively as NVAS $v$. placebo.

To test the effect of NVAS on overall cytokine responsiveness irrespective of stimulation, a collective test for each of the in vitro cytokines was performed for distributions with $<50 \%$ measurements below LLD, except for cytokines where the effect estimates across the different stimulations in the particular analysis were too heterogeneous $(P<0.05)$ to be combined.

The effect of NVAS was analysed overall and stratified by sex. 


\section{Ethics}

In both the NVAS trial and the immunological MV sub-group study, the mothers were informed by a trained field worker about the studies in their own language, Portuguese Creole, and, provided consent was given, they were asked to sign or fingerprint the consent form. The studies adhered to the Helsinki Declaration. The protocols of the respective studies were approved by the Ministry of Health in Guinea-Bissau, and the Danish Central Ethical Committee gave its consultative approval.

\section{Results}

In total, the study included 247 infants, who enrolled in both the NVAS RCT and in the immunological MV sub-group study to the early MV trial (Fig. 1). The infants included in the present analysis only represent a fraction of the participants in the NVAS trial. To assess whether a particular selection of the cohort had taken place, we therefore compared background characteristics of the herein analysed infants with the participants enrolled in the NVAS trial during the same period, but not included in the present study. There were only very subtle differences between
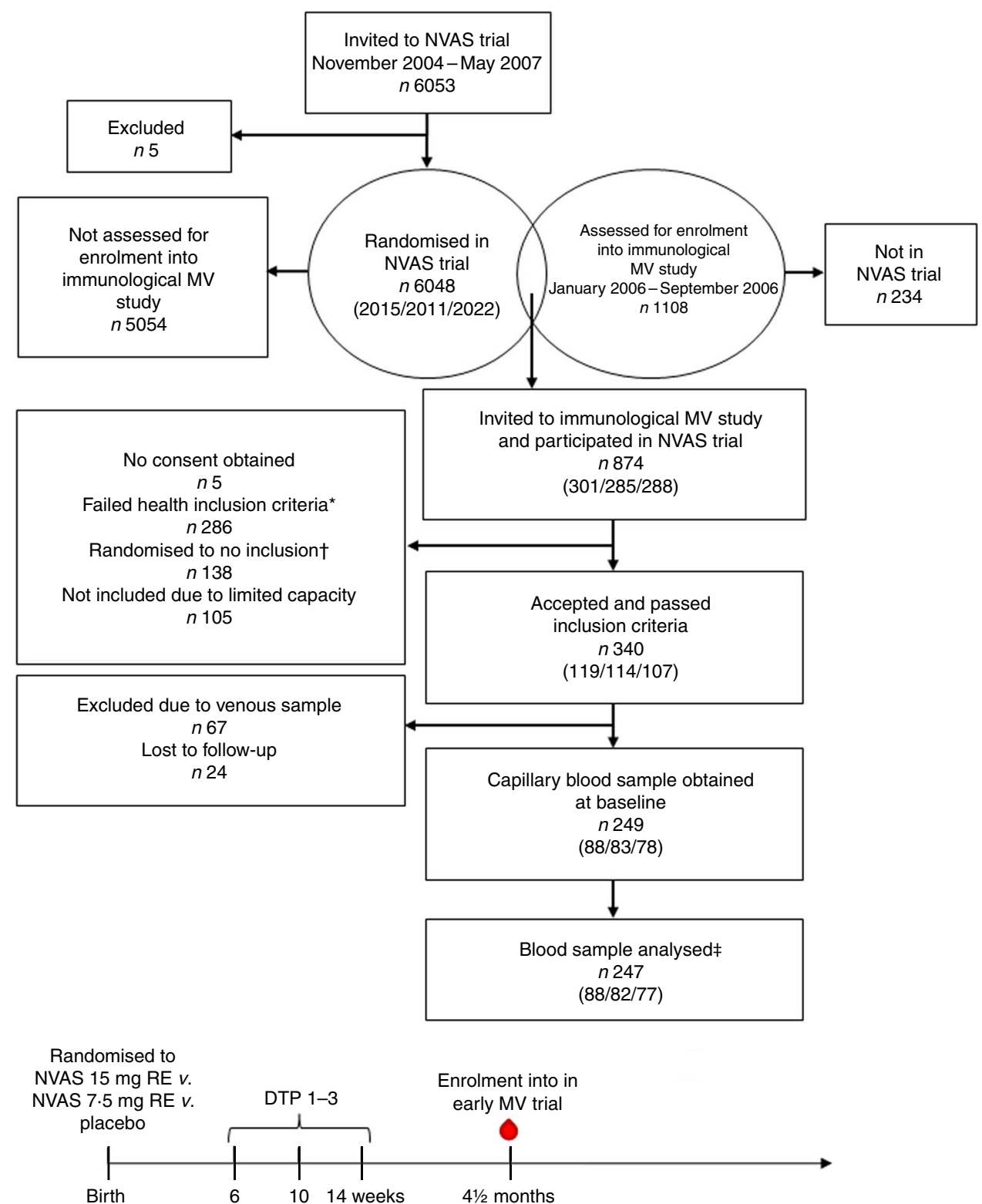

Fig. 1. Flow chart of participants and timeline of studies. Numbers in parentheses indicate the number of infants randomised to either of the three treatment arms: 30 mg retinol equivalents (RE) (50000 IU vitamin A)/7.5 mg RE (25000 IU vitamin A)/placebo. NVAS, neonatal vitamin A supplementation; MV, measles vaccine; DTP, diphtheriatetanus-pertussis vaccine; 0 , blood sample used in the present study. * Health exclusion criteria were as follows: current fever or diarrhoea reported by the mother; an axillary temperature above $37.5^{\circ} \mathrm{C}$; a respiratory rate at $60 / \mathrm{min}$ or above; or current infection diagnosed by the examining physician. $\dagger$ As the Early $\mathrm{MV}$ trial randomised infants $1: 2$ to early MV $v$. no early MV at 4.5 months of age, half of the children in the control arm of the early MV trial were randomised off inclusion to the immunological MV subgroup study, in order to achieve an equal number of infants in the immunological study in the early MV and no early MV groups, respectively. $\ddagger$ Of the total analysed blood samples, 240 had a plasma sample for ex vivo inflammatory marker analysis, and 208 had an in vitro-stimulated blood sample for cytokine response analysis. 
Table 1. Population characteristics

(Percentages and numbers; median values and 10th-90th percentiles; mean values and standard deviations)

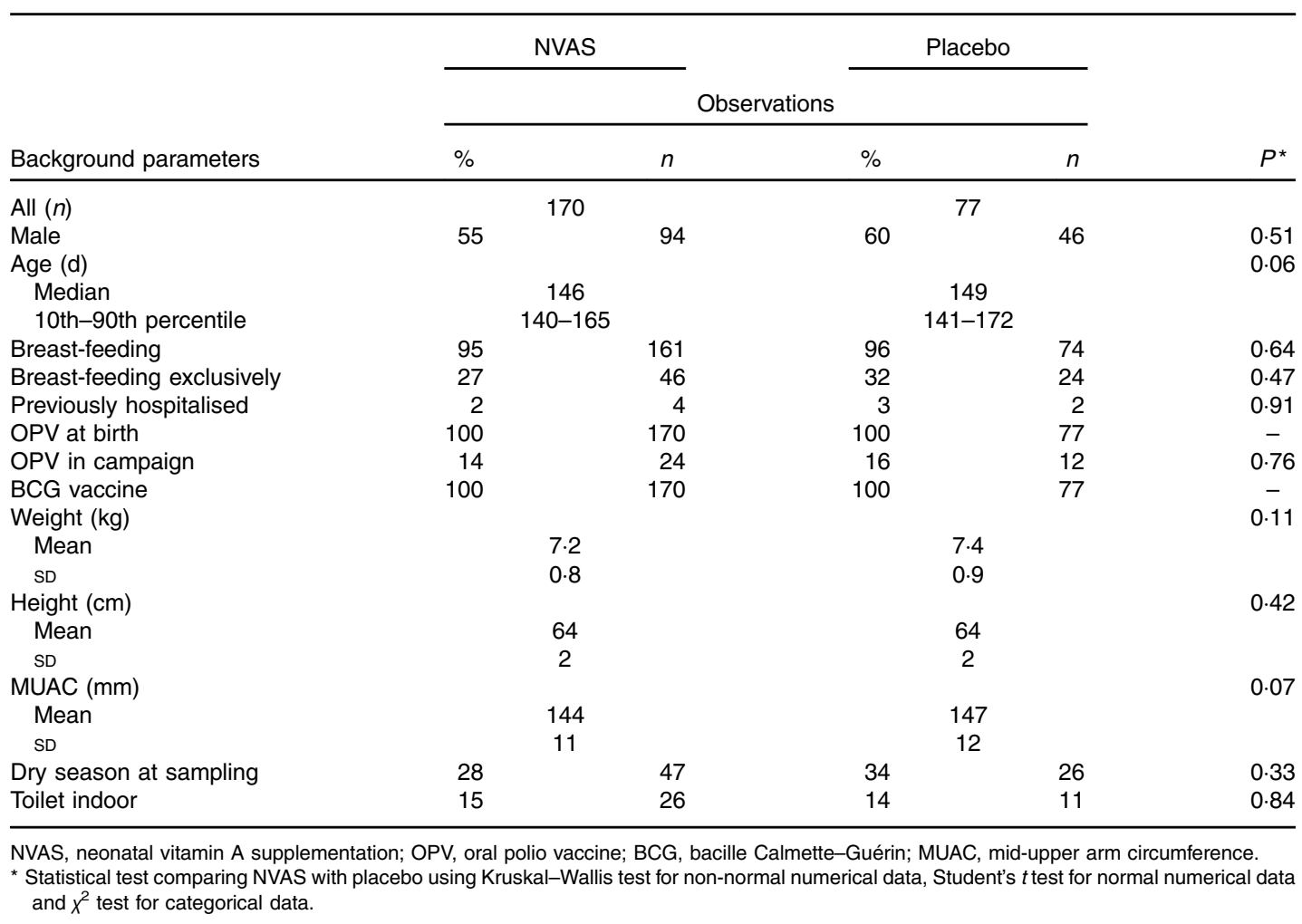

the analysed infants and the non-analysed infants, the former being slightly smaller (online Supplementary Table S2), and, importantly, of the analysed infants, the NVAS and placebo groups were similar with respect to characteristics presented in Table 1, which could influence the immune system at the age of 4-6 months (Table 1).

\section{Sex differences in the placebo group}

In the placebo group, there were strong differences between males and females. Females were less likely than males to have a BCG scar (71 v. 93\%, proportion ratio of no scar: $1.21 ; 95 \% \mathrm{CI}$ $1.05,1 \cdot 40)$. Females produced lower IFN- $\gamma$ responses and higher IL-10 responses than males did, resulting in lower pro- to anti-inflammatory TNF- $\alpha$ :IL-10 ratios and IFN- $\gamma$ :IL-10 ratios, reaching significance for the collective cytokine test (online Supplementary Fig. S1). There were no significant sex differences in plasma cytokine levels in the placebo group (data not shown), except for higher IL-1Ra levels in females (GMR: 1.19; $95 \%$ CI $1 \cdot 00-1 \cdot 40$ ).

\section{Effects of neonatal vitamin A supplementation}

Females receiving NVAS were significantly more likely to have BCG scars than the placebo group (96 v. 71\%, PR: $1 \cdot 24 ; 95 \% \mathrm{CI}$ 1.09-1.42). There was no such difference among males (PR: 1.02; $95 \%$ CI $0 \cdot 95-1 \cdot 10, P=0 \cdot 01$ for interaction between NVAS and sex).

In males, NVAS was associated with a significantly increased in vitro IL-10 to PPD (GMR: 1.99; $95 \%$ CI 1.10, 3.58), to LPS
(GMR: 1.45; $95 \%$ CI 1.02, 2.06), and for all stimulations analysed collectively, but not in females (Fig. 2). The interaction between NVAS and sex was significant for IL-10 responses analysed collectively $(P=0 \cdot 002)$. Moreover, the association of NVAS with the TNF- $\alpha$ :IL-10 ratios was sex-differential with NVAS tending to increase the rations in females, but decreasing them in males, resulting in significant interactions between NVAS and sex for PPD $(P=0.05)$, TT $(P=0.04)$, medium alone $(P=0.03)$ and for the ratios analysed collectively irrespective of the stimulation $(P=0 \cdot 02)$. A similar pattern was observed for the IFN- $\gamma:$ IL-10 and IFN- $\gamma:$ IL- 5 ratios, where NVAS was associated with reduced ratios in males exclusively, significantly so for IFN- $\gamma$ :IL-10 to PHA (GMRR NVAS-to-placebo: 0.40; 95\% CI 0.16, 0.96) and PPD (GMRR: $0 \cdot 33 ; 95 \%$ CI 0.13, 0.88), albeit the interaction with sex was not significant. In males but not in females, IL-17 responses to PHA (GMR: 1.69; 95\% CI 1.10, 2.59) and for all stimulations analysed collectively were higher in NVAS than placebo recipients (Fig. 2).

For plasma biomarkers, NVAS was associated with increased IL-1Ra in males (GMR: 1.16; $95 \%$ CI 1.01, 1.34), but not in females (GMR: 0.96; $95 \%$ CI 0.81, 1.14, $P_{\text {for interaction }}=0 \cdot 10$ ). NVAS was not associated with other plasma cytokine levels in either sex (data not shown).

\section{Discussion}

The present study indicates long-lasting effects of NVAS on responses to BCG vaccination and immunological responsiveness of peripheral blood cells persisting at 4-6 months of age in a 


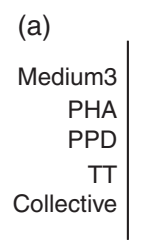

(c)

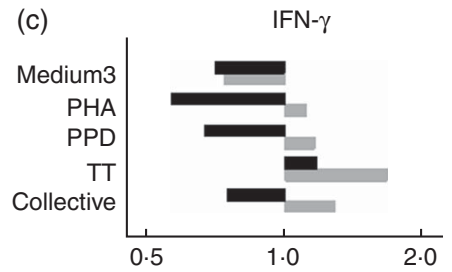

(e)

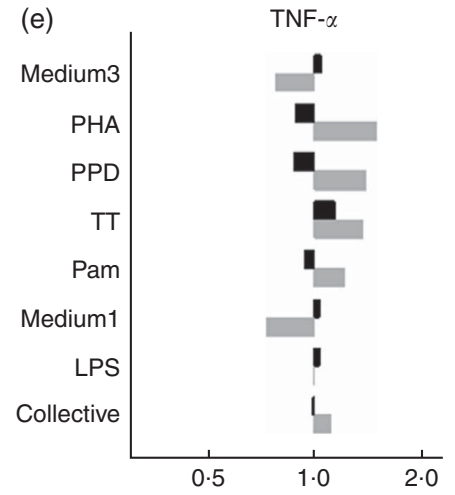

(b)

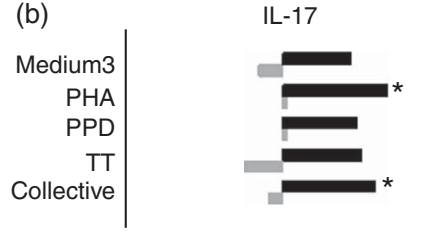

(d)
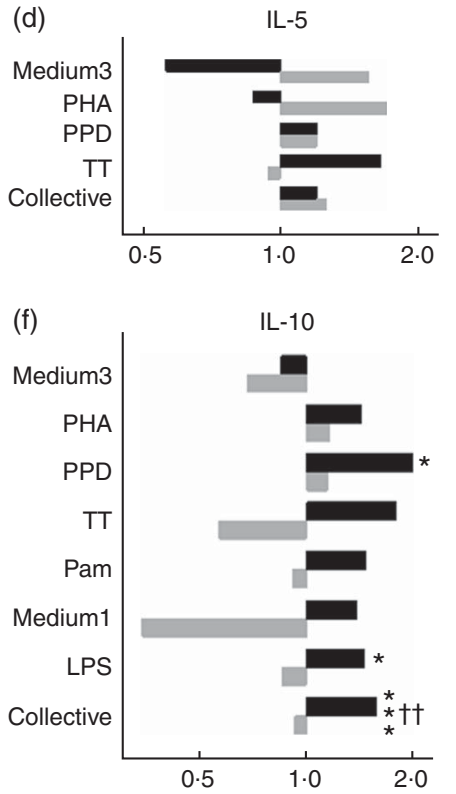

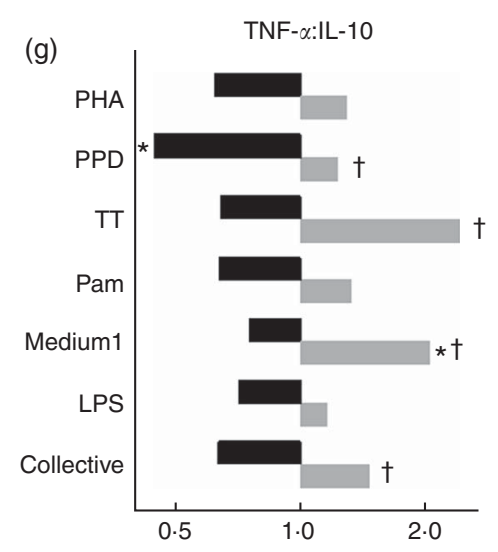

(h)

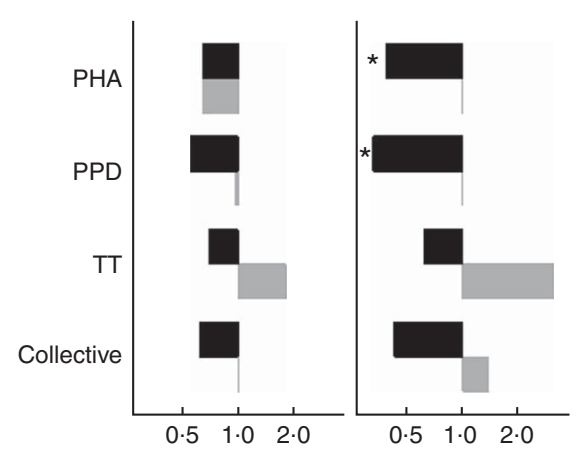

Fig. 2. Effects of neonatal vitamin A supplementation on in vitro cytokine responses at the age of $4-6$ months, stratified by sex. (a-f) Geometric mean ratios (GMR) of cytokine responses, comparing NVAS with placebo, stratified by sex. For responses of IL-13, IL-17, IL-10, IFN- $\gamma$ and IL-5 in the medium 3 condition and IL-17 to purified protein derivative from Mycobacterium tuberculosis (PPD), >50\% of measurements were below the lower detection limit; hence, these outcomes were analysed as the frequency of measureable values by Poisson regression, and reported as proportion ratios of measurements being above the lower limit. ( $g$ and $h$ ) GMR-ratios (GMRR) of cytokine responses TNF- $\alpha$ :IL-10 (g) or IFN- $\gamma: \mathrm{IL}-5$ and IFN- $\gamma: \mathrm{IL}-10$ (h), comparing NVAS with placebo; only cytokine distributions with $>50 \%$ detectable observations were included in the analysis. A GMR or GMRR $>1$ can be interpreted as an increasing effect of NVAS on cytokine concentrations or ratios, respectively. Note that the $x$-axes for IL-13, IL-17 and IFN- $\gamma$ and IL-5 are identical. Medium3, medium1: culture with medium alone for 3 or $1 \mathrm{~d}$, respectively; PHA, phytohaemagglutinin; TT, tetanus toxoid; collective: cytokine responses were analysed collectively, grouped across the different stimulations, excluding outcomes with $<50 \%$ measurements within detection ranges of the assay; Pam, palmitoyl(3)-cysteine-serine-lysine(4); LPS, lipopolysaccharide; $\square$, Males; $\square$, females. Statistical test for effect of NVAS: ${ }^{*} P<0.05 ;{ }^{\star \star *} P<0.001$; statistical test for interaction between NVAS and sex: $\dagger P<0.05 ; \dagger \dagger P<0.01$.

sex-dependent manner. NVAS $v$. placebo was associated with increased frequency of BCG scars in females, making scar frequencies in NVAS-receiving females comparable to those of boys in general. NVAS was also associated with more pro-inflammatory cytokine responses in females, whereas the opposite tendency was seen in males. Sex differences in NVAS effects were found for responses to several recall antigens and for baseline secretion in non-stimulated cells, suggesting generalised sex-dependent immune-modulatory properties of NVAS. Except for IL-1Ra, plasma inflammatory markers were not associated with NVAS, indicating that the effects observed for in vitro responses were not due to differences in systemic inflammation at the time of bleeding.

\section{Strengths and weaknesses}

The investigation of immunological effects of NVAS was conducted in a relevant cohort of infants in a low-income high disease burden setting, with a documented high proportion of individuals with low vitamin A levels ${ }^{(15)}$, and hence a potential target for VAS distribution. The data were generated in a cohort of infants previously randomised to VAS or placebo, and an overall balanced distribution of background factors remained in the present group of participants at blood sampling.

The immunological study, however, was not planned before conducting the NVAS trial; the samples were collected in connection to a subsequent immunological MV sub-group study of shorter duration and smaller size, inevitably implying that only a minor sub-group of the NVAS trial participants were followed up. Therefore, a biased selection of individuals cannot be ruled out, although the available background characteristics from the NVAS trial do not indicate any particular selection. The immunological assay was designed to explore potential non-specific immunological effects of early MV on cytokine recall responses to heterologous vaccines in addition to typical innate stimulation, investigating Th1-, Th2- and Th17-related responses. Although the assessment of the thirty different stimulationcytokine combinations entailed a considerable risk of chance findings, we did not use adjustment for multiple testing owing to the explorative nature of the study. The findings should be interpreted with this potential caveat in mind. However, the overall rather consistent sex-differential pattern of NVAS dependent cytokine responses is unlikely to be a spurious 
association owing to multiple testing. By inherent limitations of the assay, cellular sources of the measured cytokines could not be elucidated, hence hampering a mechanistic interpretation.

\section{Comparison with other studies}

Sex differences in basic immunological parameters in infancy have been reported previously, including higher NK cell counts and increased inflammatory in vitro responses to LPS and mitogen stimulation in males ${ }^{(16)}$. A recent Gambian study in a cohort where VAS is routinely given at 6 months (KL Flanagan, personal communication) found reduced type-1 T-cell reactivity in females compared with males at 10 months of age ( 1 month after DTP vaccination), as evidenced by lower IL-12p70 responses and IFN- $\gamma:$ IL- 4 ratios in unspecific T-cell stimulation ${ }^{(17)}$. This seemingly contrasts the elevated IFN- $\gamma$ :IL- 5 ratios to TT and PPD in females compared with males among the NVAS recipients in the present fully DTP vaccinated cohort. However, in likening with the DTP arm of the Gambian study, the present study found no sex differences among NVAS recipients in the TNF- $\alpha$ :IL-10 ratios to LPS, TT or PPD.

In addition to sex differences per se, sex differences in immunological effects of VAS in children have also been described, although the evidence of the effects is not unambiguous in the literature. A previous study from Guinea-Bissau reported no effect of NVAS on BCG scar frequencies at 2 and 6 months overall or by sex, as both males and females had high scar frequencies also in the placebo group. NVAS was associated with increased in vitro IFN- $\gamma$ responses to PPD in males at 6 weeks, but had no effect on IL-10 responses to PPD; NVAS was also associated with a transiently lower proportion of tuberculin skin test responders at 2 months among males, but not females ${ }^{(18)}$. Another study from Guinea-Bissau evaluating the immunological effect of NVAS 6 weeks after administration found the strongest effects of NVAS in DTP-vaccinated males. NVAS reduced TNF- $\alpha$ and IL-10 secretion in medium alone in males with prior DTP, but not in females ${ }^{(19)}$. A third immunological study from Guinea-Bissau investigating the effect of VAS $v$. placebo administered together with $\mathrm{MV}$ in >6-month-old children found that VAS tended to decrease all measured cytokine responses to PHA (TNF- $\alpha$, IL-10, IL-2, IL-5 and IFN- $\gamma$ ) in females, but not in males, six weeks after administration; this, however, was exclusively found in infants, who had not previously received $\mathrm{VAS}^{(13)}$. Overall, these previous findings are not unequivocally corroborated by the present study at 4.5 months of age, in which all infants had received three doses of DTP; herein, NVAS was associated with anti-inflammatory responses in males, but with pro-inflammatory responses in females.

Part of the discrepancy may be attributed to differences in age of VAS receipt, as VAS at birth $v$. VAS at 6 months may have different sex-dependent health effects, or differences in age of sampling and interaction with vaccination ${ }^{(20)}$. In the immunological MV sub-group study from which the present study data used the baseline samples, infants were randomised to receiving early MV or no early MV (equal distribution of NVAS and placebo infants). We have previously published that six weeks after randomisation, MV was associated with increased TNF- $\alpha$ : IL-10 ratios in infants who had previously received NVAS, whereas MV tended to decrease the ratios in children without previous NVAS. This pattern, however, was not significantly different between males and females ${ }^{(11)}$. Hence, MV may enhance the pro-inflammatory effect of NVAS in females, whereas MV may ameliorate the anti-inflammatory effect of NVAS in males. The latter would be in keeping with the Gambian infant study, in a setting where VAS is routinely distributed at 6 months, and where MV at 9 months of age was associated with enhanced pro-inflammatory marker levels in plasma and responses to TLR4 agonist and PPD stimulation in males but not in females 1 month after MV compared with the pre-MV baseline ${ }^{(17)}$

In the trial of early MV using all-cause mortality as an outcome, NVAS-receiving females tended to benefit from early MV (as compared with no early MV), whereas early MV was detrimental for NVAS-receiving males ${ }^{(10)}$. A combined analysis of three NVAS RCT in Guinea-Bissau, including the present, found that the overall mortality effect of NVAS was significantly sex-differential, with increased female mortality ${ }^{(6)}$, which may be caused by a negative interaction with $\mathrm{DTP}^{(9)}$. Hence, in females, NVAS $v$. placebo may be associated with increased pro-inflammatory responsiveness and an increased mortality risk after DTP, whereas the increased pro-inflammatory responsiveness after MV in NVAS recipients seems not to be associated with increased mortality risk in females. Further studies are needed to potentially reconcile these seemingly contrasting immunological and survival effects of NVAS and vaccines.

Interestingly, in the same setting, NVAS has also been associated with an increased risk of atopy in older children. In two follow-up studies in Bissau in children aged 3-9 years and 8-10 years who previously enrolled in trials of VAS at birth (in lowbirth weight and normal-birth weight infants, respectively), NVAS $v$. placebo was associated with increased risk of atopy (skin prick test) and wheezing, although in the latter study this association was exclusively seen in females ${ }^{(21,22)}$. Studies on the potential associations between cytokine responses and subsequent risk for development of atopic diseases are not unambiguous; however, a large body of evidence indicates that Th2-biased and IFN- $\gamma$-reduced cytokine response profiles in cord blood and infancy are be associated with increased risk of development of atopy in childhood (e.g. ${ }^{(23,24)}$ ); for example, in a prospective cohort study, decreased IFN- $\gamma$ :IL- 4 and IFN- $\gamma$ :IL-10 response ratios of PMA/ionomycin-stimulated PBMC in very young infants with eczema were associated with increased risk of asthma at 4 years of age ${ }^{(25)}$. In the present cytokine study, NVAS tended to drive cytokine responses towards a Th2-bias, but in males only, potentially suggesting sex-differential associations between NVAS, cytokine patterns and atopic disease.

In conclusion, the present study corroborates sex-differential effects of VAS on the immune system, finding that NVAS was associated with an increased scar formation after BCG vaccination and increased pro- to- anti-inflammatory cytokine response ratios in females, but not in males, emphasising the importance of analysing VAS effects by sex. 


\section{Acknowledgements}

The authors thank Jesper Eugen-Olsen for suPAR analysis (suPARnostic standard ELISA, ViroGates, Denmark).

The present study received support from Novo Nordisk Foundation, Fonden til Lægevidenskabens Fremme, Danish Medical Research Council, Augustinus Fonden, Beckett fonden, Dagmar Marshalls fond and the Aase og Ejnar Danielsens fond. P. A. holds a research professorship grant from the Novo Nordisk Foundation. C. S. B. is funded by an European Research Council (ERC) Starting Grant (ERC-2009-StG-243149). Research Center for Vitamins and Vaccines (CVIVA) is funded by the Danish National Research Foundation (DNRF108). The funders had no role in study design, data collection and analysis, decision to publish or preparation of the manuscript.

K. J. J. analysed the data and wrote the manuscript; M. J. S. and C. M. supervised the field work and collection of blood samples; M. J. S. handled blood samples in the laboratory and performed in vitro stimulations; A. A. supervised data analysis; P. A. and C. S. B. conceived the research idea and designed the randomised trials; C. E., C. S. B. and M. J. S. designed the immunological study. All authors read and approved the final version of the manuscript.

None of the authors has any conflicts of interest to declare.

\section{Supplementary material}

For supplementary material/s referred to in this article, please visit https://doi.org/10.1017/S0007114517002938

\section{References}

1. Raverdeau M \& Mills KH (2014) Modulation of T cell and innate immune responses by retinoic acid. J Immunol 192, 2953-2958.

2. World Health Organization (2011) Guideline: Vitamin A Supplementation in Infants and Children 6-59 Months of Age. Geneva: WHO.

3. Haider BA \& Bhutta ZA (2015) Neonatal vitamin A supplementation: time to move on. Lancet 385, 1268-1271.

4. World Health Organization (2011) Guideline: Neonatal Vitamin A Supplementation. Geneva: WHO.

5. Benn CS, Diness BR, Roth A, et al. (2008) Effect of 50,000 IU vitamin A given with BCG vaccine on mortality in infants in Guinea-Bissau: randomised placebo controlled trial. BMJ 336, 1416-1420.

6. Benn CS, Diness BR, Balde I, et al. (2014) Two different doses of supplemental vitamin A did not affect mortality of normalbirth-weight neonates in Guinea-Bissau in a randomized controlled trial. J Nutr 144, 1474-1479.

7. Benn CS, Fisker AB, Napirna BM, et al. (2010) Vitamin A supplementation and BCG vaccination at birth in low birthweight neonates: two by two factorial randomised controlled trial. BMJ 340, c1101.

8. Benn CS, Aaby P \& Fisker AB (2015) Neonatal vitamin A: time to move on? Lancet 386, 132-133.

9. Benn CS, Rodrigues A, Yazdanbakhsh M, et al. (2009) The effect of high-dose vitamin A supplementation administered with BCG vaccine at birth may be modified by subsequent DTP vaccination. Vaccine 27, 2891-2898.

10. Aaby P, Martins CL, Garly ML, et al. (2010) Non-specific effects of standard measles vaccine at 4.5 and 9 months of age on childhood mortality: randomised controlled trial. BMJ 341, c6495.

11. Jensen KJ, Sondergaard M, Andersen A, et al. (2014) A randomized trial of an early measles vaccine at 4(1/2) months of age in Guinea-Bissau: sex-differential immunological effects. PLOS ONE 9, e97536.

12. Eriksson M, Sartono E, Martins CL, et al. (2007) A comparison of ex vivo cytokine production in venous and capillary blood. Clin Exp Immunol 150, 469-476.

13. Jensen KJ, Fisker AB, Andersen A, et al. (2016) The effects of vitamin A supplementation with measles vaccine on leucocyte counts and in vitro cytokine production. BrJ Nutr 115, 619-628.

14. Jensen KJ, Larsen N, Biering-Sorensen S, et al. (2014) Heterologous immunological effects of early BCG vaccination in low-birth weight infants in Guinea-Bissau: a randomizedcontrolled trial. J Infect Dis 211, 956-967.

15. Danneskiold-Samsoe N, Fisker AB, Jorgensen MJ, et al. (2013) Determinants of vitamin A deficiency in children between 6 months and 2 years of age in Guinea-Bissau. BMC Public Health 13, 172.

16. Klein SL \& Flanagan KL (2016) Sex differences in immune responses. Nat Rev Immunol 16, 626-638.

17. Noho-Konteh F, Adetifa JU, Cox M, et al. (2016) Sexdifferential non-vaccine-specific immunological effects of diphtheria-tetanus-pertussis and measles vaccination. Clin Infect Dis 63, 1213-1226.

18. Diness BR, Fisker AB, Roth A, et al. (2007) Effect of highdose vitamin A supplementation on the immune response to Bacille Calmette-Guerin vaccine. Am J Clin Nutr 86, 1152-1159.

19. Jorgensen MJ, Fisker AB, Sartono E, et al. (2013) The effect of at-birth vitamin A supplementation on differential leucocyte counts and in vitro cytokine production: an immunological study nested within a randomised trial in Guinea-Bissau. Br J Nutr 109, 467-477.

20. Benn CS, Aaby P, Arts RJ, et al. (2015) An enigma: why vitamin A supplementation does not always reduce mortality even though vitamin A deficiency is associated with increased mortality. Int J Epidemiol 44, 906-918.

21. Kiraly N, Benn CS, Biering-Sorensen S, et al. (2013) Vitamin A supplementation and BCG vaccination at birth may affect atopy in childhood: long-term follow-up of a randomized controlled trial. Allergy $\mathbf{6 8}, 1168-1176$.

22. Aage S, Kiraly N, Da CK, et al. (2015) Neonatal vitamin A supplementation associated with increased atopy in girls. Allergy 70, 985-994.

23. Allam JP, Zivanovic O, Berg C, et al. (2005) In search for predictive factors for atopy in human cord blood. Allergy 60, 743-750.

24. Stern DA, Guerra S, Halonen M, et al. (2007) Low IFN-gamma production in the first year of life as a predictor of wheeze during childhood. I Allergy Clin Immunol 120, $835-841$.

25. Sarria EE, Mattiello R, Yao W, et al. (2014) Atopy, cytokine production, and airway reactivity as predictors of pre-school asthma and airway responsiveness. Pediatr Pulmonol 49, 132-139. 